EDITORIAL

\title{
Faricimab phase 3 DME trial significance of personalized treatment intervals (PTI) regime for future DME trials
}

(c) The Author(s), under exclusive licence to The Royal College of Ophthalmologists 2021

Eye (2022) 36:679-680; https://doi.org/10.1038/s41433-021-01831-4

Faricimab is the first bispecific antibody designed for the eye. It acts on two pathways - the angiopoietin-2 (Ang-2) and vascular endothelial growth factor-A (VEGF-A). Ang-2 and VEGF-A are vessel-destabilizing factors that promote the formation of new leaky blood vessels, leading to increased vascular permeability and inflammation [1]. Our group has previously highlighted how the preclinical and phase I/II data have shown efficacy and safety of the molecule with extended durability and the potential for up to 16-week dosing intervals [2]. Recently, Roche (Basel, Switzerland) released results of four phase III studies of faricimab, two for the treatment of diabetic macular oedema (DME) and two for the treatment of neovascular age-related macular degeneration (nAMD) [3]. Faricimab is the first injectable ophthalmic drug to achieve up to 16 weeks extended treatment interval in phase III studies for DME and nAMD. Faricimab was well-tolerated in these trials with no new or unexpected safety signals.

Faricimab has an interesting phase 3 trial design, which is different in DME compared to nAMD. In nAMD, faricimab was dosed at fixed intervals of every 2, 3, or 4 months based on the disease activity at weeks 20 and 24 after a monthly loading phase. However, DME trial had an arm titled personalized treatment interval (PTI) apart from the fixed every other month treatment arm after respective monthly loading phases.

PTI regimen is novel as it allows for a change in the frequency of injection from 1 month to 4 months, up and down depending on the activity of the disease. Such flexible injection frequency has never been tested in the pivotal clinical trials, which were used for the approval of other anti-VEGFs for DME approval. Ranibizumab used a fixed monthly regimen in RIDE and RISE trial [4] and aflibercept had fixed monthly and bimonthly treatment arms after 5 monthly injections in the VISTA and VIVID trials [5]. The probable reason was the pharmacokinetics of these drugs that allowed for a maximum extension of up to 8 weeks with aflibercept. With the availability of newer drugs that may have a longer duration of action such as brolucizumab and faricimab, having a PTI type of regimen in the trial arm might be a better way to determine clinical durability. Brolucizumab has submitted the application for the United States food and drug administration (US-FDA) approval in DME on the basis of the phase 3 KITE and KESTREL trial results. Although dosing was adaptive in nature, given in a fixed fashion either 2 or 3 months based on disease activity after the loading phase, it was not individualized by lengthening or shortening the interval as was the case in the PTI regime of phase 3 DME studies with faricimab, which is more akin to a treat-and-extend (T\&E) regimen utilized in clinical practice [6].

The strength of the PTI regimen is its simulation of real-world practice. We have seen in the past that patients in the real world are often not treated like in the trials and this may be a contributing factor to the discrepancy between the trial and the real-world outcomes with results typically favoring the former. PTI is an important regimen for the phase 3 trial in DME as diabetes is a systemic disease and diabetic retinopathy with its complications such as DME depends on the systemic disease control in contrast to $\mathrm{nAMD}$.

A limitation of PTI is that the variable and reactive dosing style may undermine the true potential efficacy of the drug and may allow for more fluctuation in the central foveal thickness which may not be considered to be optimal disease control in the long term, as per the recent evidence. To overcome these limitations, YOSEMITE and RHINE trials included a fixed regimen with an every other month faricimab treatment arm.

In the YOSEMITE trial, for faricimab, the average visual gains were +11.6 and +10.7 letters in the PTI and every other month arms, respectively, and +10.9 letters in the aflibercept arm. In RHINE, for faricimab, the average visual gains were +10.8 and +11.8 letters in the PTI and every other month arms, respectively, and +10.3 letters in the aflibercept arm. Both the studies measured the proportion of people in the faricimab PTI arm that achieved dosing schedules every 3 or 4 months at the end of the first year as the secondary endpoint assessment criteria. 52.8\% $(n=151 / 286)$ of faricimab PTI patients in YOSEMITE and 51\% $(n=157 / 308)$ in RHINE achieved 4-month dosing at 1 year and $21 \%(n=60 / 286)$ of faricimab PTI patients in YOSEMITE and $20.1 \%(n=62 / 308)$ in RHINE achieved 3 -month dosing. Hence, $>70 \%$ of patients on the faricimab PTI regimen were able to have 3 months or longer treatment intervals at the end of the first year.

There are various treatment regimens such as monthly T\&E and pro re nata that are currently in practice for the management of nAMD. However, there has been a lack of consensus on the ideal treatment regimen for the management of DME. Our group had proposed a regimen entitled SNF-Ob (Subfoveal Neurosensory Flattening and Observe) that has the potential to reduce the injection burden in a specific subgroup of DME with neurosensory detachment [7]. However, it needs further validation to implement it on a larger scale and we are in the process of validating this regime with a larger database.

To summarize, the inclusion of an individualized PTI regimen in phase 3 faricimab trial might pave way for future trials to better understand drug durability and injection interval in the clinical practice and also the possibility of reducing the injection burden. Progress from the monthly regimen used during the ranibizumab phase 3 trials to the PTI regime for faricimab is a potentially valuable clinical trial design because it is very similar to the popular T\&E regimen. Phase 3 trial designs that use PTI for anti-VEGF agents, whether it be for nAMD or DME, may better reflect the real-world practice of drugs after approval once commercialized. 
Ashish Sharma $\mathbb{D}^{1 凶}$, Nilesh Kumar $\mathbb{D D}^{1,2}$, Nikulaa Parachuri ${ }^{1,3}$, Dhatri Karanam ${ }^{1}$, Baruch D. Kuppermann ${ }^{\prime}$, Francesco Bandello iD ${ }^{5}$ and Carl D. Regillo ${ }^{6}$

${ }^{1}$ Lotus Eye Hospital and Institute, Avinashi Road, Coimbatore, TN, India. ${ }^{2}$ Madhavi Netralaya, Ara, Bihar, India. ${ }^{3}$ Department of

Vitreoretina, Sankara Eye Hospital, Coimbatore, $T N$, India. ${ }^{4}$ Gavin Herbert Eye Institute, University of California, Irvine, Irvine, CA, USA. ${ }^{5}$ University Vita-Salute, Scientific Institute San Raffaele, Milano, Italy. ${ }^{6}$ The Retina Service of Wills Eye Hospital, Mid Atlantic Retina, Philadelphia, PA, USA. ${ }^{凶}$ email: drashish79@hotmail.com

\section{REFERENCES}

1. Sharma A, Kumar N, Parachuri N, Bandello F, Kuppermann BD, Loewenstein A. Faricimab: two in the bush is proving better than one in the hand? Ocul Immunol Inflamm. 2021;9:1-3.

2. Sharma A, Kumar N, Kuppermann BD, Bandello F, Loewenstein A. Faricimab: expanding horizon beyond VEGF. Eye. 2020;34:802-4.

3. New phase III data show Roche's faricimab is the first investigational injectable eye medicine to extend time between treatments up to four months in two leading causes of vision loss, potentially reducing treatment burden for patients. https:// www.roche.com/media/releases/med-cor-2021-02-12.htm\#: :text=Combined\%2C \%20more\%20than\%2070\%25\%20of,aflibercept\%20given\%20every\%20two\% 20months. 2021.

4. Nguyen QD, Brown DM, Marcus DM, Boyer DS, Patel S, Feiner L.RISE and RIDE Research Group et al. Ranibizumab for diabetic macular edema: results from 2 phase III randomized trials: RISE and RIDE. Ophthalmology. 2012;119:789-801.

5. Heier JS, Korobelnik JF, Brown DM, Schmidt-Erfurth U, Do DV, Midena E, et al. Intravitreal aflibercept for diabetic macular edema: 148-week results from the VISTA and VIVID studies. Ophthalmology. 2016;123:2376-85.

6. Novartis Phase III Beovu ${ }^{\circledR}$ data show potential for fluid resolution in more diabetic macular edema patients with fewer injections versus aflibercept. https://www. novartis.com/news/media-releases/novartis-phase-iii-beovu-data-show-potentialfluid-resolution-more-diabetic-macular-edema-patients-fewer-injections-versusaflibercept. Accessed Aug 06, 2021

7. Sharma A, Parachuri N, Kumar N, Kuppermann BD, Bandello F, Loewenstein A, et al. Subfoveal Neurosensory Detachment Flattening and Observe (SNF-Ob): a novel approach in diabetic macular edema management: a potential cost-effective treatment strategy to be explored. Ophthalmol Retin. 2019;3:1009-1011.

\section{ACKNOWLEDGEMENTS}

Dr. Kuppermann acknowledges an unrestricted grant from Research to Prevent Blindness to the Gavin Herbert Eye Institute at the University of California, Irvine.

\section{AUTHOR CONTRIBUTIONS}

A.S.: conception, analysis, drafting, integrity check, final approval. N.P., N.K., D.K., B.D.K., F.B., C.R.: drafting, revision, analysis, integrity check.

\section{COMPETING INTERESTS}

Dr. Ashish Sharma: consultant for Novartis, Allergan, Bayer, and Intas. Baruch D. Kuppermann: clinical research: Alcon, Alimera, Allegro, Allergan, Apellis, Clearside, Genentech, GSK, lonis, jCyte, Novartis, Regeneron, ThromboGenics; consultant: Alimera, Allegro, Allergan, Cell Care, Dose, Eyedaptic, Galimedix, Genentech, Glaukos, Interface Biologics, jCyte, Novartis, Ophthotech, Regeneron, Revana, Theravance Biopharma. Dr. Francesco Bandello: consultant: Allergan, Bayer, BoehringerIngelheim, FidiaSooft, Hofmann La Roche, Novartis, NTC Pharma, Sifi, Thrombogenics, Zeiss. Dr. Carl D Regillo: consultant: Allergan, Chengdu Kanghong, Genentech/Roche, Novartis, Kodiak, Notal, Merck, Shire-Takeda, Adverum, Graybug, and Eyepoint and receives research support from Allergan, Chengdu Kanghong, Genentech/Roche, Novartis, Kodiak, Iveric, and Adverum. Dr. Nilesh Kumar, Dr. Nikulaa Parachuri, and Dr. Dhatri Karanam: none.

\section{ADDITIONAL INFORMATION}

Correspondence and requests for materials should be addressed to Ashish Sharma.

Reprints and permission information is available at http://www.nature.com/ reprints

Publisher's note Springer Nature remains neutral with regard to jurisdictional claims in published maps and institutional affiliations. 